\title{
High-precision and cost-efficient sequencing for real-time COVID-19 surveillance
}

\section{Sung Yong Park \\ Gina Faraci \\ Pamela M. Ward \\ Jane F. Emerson \\ Ha Youn Lee}

\section{Video Byte}

Keywords: COVID-19, SARS-CoV-2, genomic surveillance, disease outbreaks, whole genome sequencing, public health, CorvGenSurv, viral RNA, RNA amplification, long-read sequencing, whole genome sequencing

Posted Date: October 13th, 2021

DOl: https://doi.org/10.21203/rs.3.rs-966197/v1

License: (c) (i) This work is licensed under a Creative Commons Attribution 4.0 International License. Read Full License 


\section{Abstract}

Within its first several months, the COVID-19 pandemic had already affected more than 33 million people and claimed more than 1 million lives. While vaccines are helping slow the spread of the disease. transmission rates in some areas remain high. And the rise of new viral mutations means continued vigilance is required. Unfortunately, current genome sequencing methods are lacking, with none managing to combine high precision, a simple workflow, and low cost in one tool. The recently developed Coronavirus Genomic Surveillance (CorvGenSurv) platform is designed to close that gap. CorvGenSurv amplifies viral RNA samples and sequences them in three segments via long-read, high-throughput sequencing. This design reduces sequencing data waste, thus preventing dropouts in genome coverage, and the long-read approach makes the sequencing workflow simpler than short-read sequencing. To evaluate the platform, researchers tested nasopharyngeal/oropharyngeal swab samples collected from 25 US patients with COVID-19 in the summer of 2020. CorvGenSurv produced near full-length wholegenome sequences that revealed new mutations in five viral proteins, each of which could make the coronavirus stronger against immune attack. CorvGenSurv offers a readily adaptable design that allows for rapid response to sudden public health threats and could help officials closely monitor the evolution of COVID-19 in real time. 